\title{
A Comparative Study of Chinese Sanda and Western Kickboxing from the Perspective of Sports Culture
}

\author{
Xin Chen \\ School of Physical Education \\ Southwest University \\ Chongqing, China 400715
}

\begin{abstract}
There are differences between the Kickboxing and Chinese Sanda in the spiritual culture level, the material institutional culture level and the behavioral institutional culture level. After the comparative analysis of the various differences of these two in aspects like origin and development, cultural basis, national characteristics, protective clothing, venue setting, fighting technology and competition rules, Chinese Sanda should absorb and learn from the essence of the kickboxing technique, further enrich Chinese Sanda's technology in order to better embody Chinese martial arts' attack and defense techniques; Properly adjust Sanda's rules, and reform its protective gear, clothing, and venue; Embody the national characteristics of Chinese Sanda, further tap and highlight the essence of Chinese martial arts and better reflect the cultural characteristics and spirit of Chinese martial arts.
\end{abstract}

Keywords-Kickboxing; Chinese Sanda; sports culture

\section{INTRODUCTION}

Sports culture is a kind of culture that takes physical activity as the form of expression; it has a rich national cultural heritage and gradually became a tangible and explicit national cultural symbol in the history of culture. When comparing a sports culture with another sports culture, according to the structure of the culture, it is divided into spiritual culture level, material institutional culture level, and behavioral institutional culture level.

\section{COMPARISON ON SPIRITUAL CULTURE LEVEL}

\section{A. The Origin and Development of Kickboxing and Chinese Sanda}

Kickboxing, also known as international kickboxing, Europe and the United States full contact free karate and so on. It originated in Europe and the United States in the 1960s, the United States is the main origin and development center. With the continuous development of the times, in the historical context of global culture, economy and other large-scale exchanges, collisions, opening and integration, kickboxing gradually bred and developed. It is a kind of Western free-style full-contact freehand attack-defense fighting technique with no routines, no denominations, emphasizing individual styles, and fighting for victory. It is unique in today's boxing world, and is the best combination of modern Eastern and Western martial arts culture and techniques. It embodies the fruits and crystallization of human martial arts integration. Kickboxing is not limited to any kind of fixed routines; it advocates free play in the fighting according to the actual situation. It flexibly displays various three-dimensional offensive and defensive techniques such as punches, legs, throws, elbows, knees, and anti-joint techniques in order to eventually knock down or beat the opponent.

Chinese Sanda, commonly known as "Sanshou", in ancient it was called "Xiang bo", "Shou bo", and "Bai da". Its origin can be traced back to the production and life of our country's ancestors in the ancient times. It was formed in the Spring and Autumn Period, and flourished in the Song and Yuan Dynasties. In history, there were activities called "Chun qiu jiao shi" and "Lu tai zheng jiao". Although this can be said to have opened a precedent for Chinese Sanda, in the end it can only be called antagonistic freehand combat. In 1933, the Central National Martial Arts Museum held "The Second National Martial Arts Competition" in Nanjing, it had set up men's and women's Sanda competitions and the one who get the highest points is the winner. At this point, all attacks on other's effective positions by hand, elbow, foot, and knee will get one point ; all attacks on other's eyes, throats, and shins are fouls; Two of three rounds, such define and implement of these rules means the prototype of Sanda sports has been basically established. The real significance of modern Sanda's development in an all-round way was after 1979, the State Sports Commission conducted a planned and organized trial Sanda competition on the basis approach of "active and secure". This allowed Chinese Sanda to enter the real competition stage from the folk exercise. After 10 years of experimentation, the various martial arts schools learn from other's strong points to offset one's weakness, and build a set of Sanda techniques with Chinese characteristics on the basis of inheriting the outstanding traditional Sanda and the modern scientific training method. The Sanda techniques are basically tend to be the same, and are obtained a leap-forward development.

In 1989, Sanda was listed as a formal competition project by the State Sports Commission. Sanda became an official event for Asian Championships, World Championships, National Games and Asian Games. Since its official establishment in 1989, Sanda has developed rapidly in just a few decades and is increasingly favored by a large number of martial arts enthusiasts. In recent years, the martial arts Sanda 
has developed rapidly in the market, especially the "Chinese Martial Arts Sanda King Contests", "Chinese Sanda and American Boxing Match Play" and "China-Thai Match Play" and other large-scale events has greatly promoted the marketization of Sanda Competition, and the number of on-site audience and TV viewers goes straight up.

\section{B. The Cultural Foundation of Kickboxing and Chinese Sanda's Development}

Kickboxing broke the boundary of faction. All groups or individuals who recognize their rules of competition can participate in the kickboxing competition, which objectively provides conditions for the exchange of various types of martial arts in the world and the testing and improvement of kickboxing technique system. Therefore, the kickboxing competition system is considered as the ultimate mode of martial arts fighting by the international Martial Arts world, and is regarded as the only fair way to truly experience the strengths and weaknesses of martial arts and the strength of the players, so that the kickboxing competition rules have become the popular and recognized standard of international martial arts competition. .

China Sanda has grown up in the large environment of Eastern culture. Eastern culture pays more attention to internal, overall, macroscopical and common things, and is highly closed. Sanda is deeply influenced by Chinese traditional philosophy and takes the "Dao" as the highest realm to pursue. The norms which takes "harmony" as the core have constitute the side of Sanda which pursue stability and overall in the dynamics; the changes of "yin and yang" and "five elements" also formed the side which pursue dynamics and changes in overall and stability. Under the influence of traditional ethics, it was decided that martial arts should be preceded by "virtue" and advocating use skill to conquer the strong and heavy. This determined that the purpose to practice Sanda was not to be fight hard in brave, but to work together with "learning the words" to finally complete the pursuit of ideal personality.

\section{The National Characteristics of Kickboxing and Chinese Sanda}

The core and soul of kickboxing is competition, and defeating an opponent is its immediate goal. Thrilling, stimulating, and utilitarian are its inherent pursuits. The kickboxing is filled with admiration for strength and eulogies for competition, because Western peoples have advocated the freedom of individuality, the pursuit of individual liberation, and personal realization since ancient Greece. The Western people regard the kickboxing as an important way to achieve self-worth in order to prove the value of self-existence. This has also made the kickboxing a very rapid development and the level of skills and tactics of participating athletes has increased dramatically.

The Sanda Movement is an important component of traditional Chinese national sports and martial arts. It is gradually developed under China's specific social and historical conditions. Therefore, it has distinctive national characteristics. China has always been a state of etiquette and it is wisdom is over brave. However, as a traditional national culture, Sanda must also contain this quaint ideology. The Sanda movement also precisely reflects such cultural connotation. It requires both sides of the game must bring necessary protective gear during the game; Attack can not continued after other side is knocked down or fell; Strictly limit the technical movements and banned parts; The winning and negative judgments mainly based on the points, do not emphasize the submission Or KO the counterpart. These also show that the Sanda movement is actually a movement that advocates the ethics of martial arts and focuses on skills; it pursues "use softness to overcome hardness".

\section{COMPARISON OF MATERIAl CUlture LEVEL}

\section{A. The Protection Clothing of Kickboxing and Chinese Sanda}

Due to the commercialization, professionalism, and openness of the Kickboxing competition, almost all international Kickboxing competitions use the "unrestricted free fighting" competition method, without restricting the qualifications of the contestants, and competing rules are similar with Thai style competitions. Both contestants are topless, wearing shorts, gloves or hand gloves for full-contact fights.

Sanda athletes must wear gloves, head guards, mouth guards, chest guards, shin guards, leg guards, bare feet, or wear foot protections as designated by the conference. They should wear vests and shorts which are the same color as the protective gears, shin guards must be worn inside shorts. Adult championships and some invitational tournaments cancelled all the protective hears besides gloves, mouth guards, and shin guards, but youth championships still retain all protective gears.

\section{B. Venues for Kickboxing and Chinese Sanda}

The venues layout of Kickboxing separated the performance area and the boxing area. This way can help overcome the shortcomings of monotony and lack of background changes during the game. A large 50-meter-wide passageway was used between the performance area and the boxing area so that before the athletes were on the field, audience could make full use of the live visuals and the closeup from the big screen to judge the athletes' mentality and strength, observe the appearance of the athletes, and determine which one to favor. The boxing field of kickboxing competition was made with 100 by $120 \mathrm{~cm}$. It is said that there are two reasons for this: First, by raising the height of the boxing field, the audience is more comfortable to see, especially the back row audience is not easily blocked by the front row audience. Second, it is convenient for television broadcasts and photo reporters to take pictures.

The venue for Chinese Sanda competition is a wooden platform with a height of $60 \mathrm{~cm}$, a length of $800 \mathrm{~cm}$ and a width of $800 \mathrm{~cm}$. On the platform is covered with cushion, canvas is covered on the cushion, at the center is painted with a yin and yang Tai Chi diagram with a diameter of $100 \mathrm{~cm}$ or with the game related patterns. There are $5 \mathrm{~cm}$ wide red borders lines on the edge of the platform. The countertops are painted with a $10 \mathrm{~cm}$ wide yellow cordon at $90 \mathrm{~cm}$ inwards. 
There are protective cushions 20 - 40 centimeters thick and $200 \mathrm{~cm}$ wide around the platform.

\section{COMPARISON ON INSTITUTIONAL CULTURE LEVEL}

\section{A. Technical Comparison}

Kickboxing is not rigidly adhere to any fixed routine moves, but to promote free play in combat according to the actual situation, flexible display fist, feet, elbow, knee, fall and other three-dimensional techniques, with both long and short attacks and make full application in order to knock down or defeat opponents. "Tell me the basic principle; I will come up with a unique technique that suits me". This is the best general expression of the concept of kickboxing. Under the guidance of this ideology, after decades of practical exploration and learning from the various martial arts schools in the world, kickboxing has formed a fairly complete boxing theory and technical tactics system as well as competition rules, under the conditions of international martial arts competitions, with its distinctive combat function and commercial operation mode, it has made remarkable achievements and has occupied an important place in the international martial arts field.

Chinese Sanda is a technique that focuses on "kickingfar, hit close, and close-touch fall" in Chinese traditional martial arts. It is manifested by various Fists, leg techniques, and wrestling. Sanda technology is based on the partial integration of traditional Chinese attack techniques and has been continuously integrated and developed. The Xiapi Quan in Chinese martial arts is reflected in the Xingyi Quan, Nan Quan, Taiji Quan and other big or small boxing schools, but it is barely used in Sanda competitions. The essence of Chinese martial arts --- grappling technique has also been ruthlessly banned by the rules. It is not difficult to see that there is indeed incompleteness and closure in Chinese Sanda technology. In addition, since Sanda became an official event for a short period of time, some techniques are still affected by the traditional advantaged boxing events such as straight, pendulum, and hook in boxing just change their name as Chong, Guan, Chaoin Sanda. In Sanda the only difference from boxing is the whipping fist, and the use of whipping fist in the competition is very low. Sanda technology focuses on skills rather than knocking down, it can be seen from Sanda's wrestling technique. There is a 3 seconds limitation in Sanda's wrestling rule, clinch for more than three seconds, the referee will pause the competition, separate the players, and restart the competition. Moreover, the rules stipulate that as long as any body part other than the two feet touches the ground, it will be count as fall to the ground. After one falls to the ground, the other side will not be allowed to attack again. Therefore, most of Sanda's wrestling methods are very clever fast wrestling with legs-contact or close-contact.

\section{B. Comparison on Competition Rules}

Rules for kickboxing: 3 rounds per game, 3 minutes per round, and 2 minutes break between each round. Any player who hits, kicks or knee, elbow hits the opponent, or weakens the opponent's ability with any action and without break any rules will score. Each round any player wins 5 points will count as winner. The player who gets the highest score in the 3 rounds is the winner. In each round when the player is knocked down, fell to one's knees, or stands upright but cannot continue the game, it will count as defeated. The opponent was knocked down by the number of notaries to "10" and could no longer stand up will be counted as defeated. Wherever the player is hit outside the arena, it is negative 1 point. From the number of notaries to "10", they failed to return to arena in time then it will be counted as defeated. The bell sounded to indicate the start of a round, and players who could not continue to play will be counted as defeated. Kickboxing fouls: Anyone who commits any of the following moves is guilty of foul theory. After the opponent is knocked down, he or she continues to whip, throw, bump or spitting, bite, kick, etc to the other player. Insert other's eyes. Use grappling techniques to reverse other's joints; attack groin, lock other's neck.

The rules of Chinese Sanda Competition have been continuously improved. The current rules are based on the "Martial Arts Sanda Competition Rules" (trial version) in 2004 The nature of Sanda's competition can include group competitions and individual competitions. Sanda competition methods can be followed as round robin, single defeat elimination, double defeat elimination. The official Sanda competitions use Best of three rounds, 3 minutes per round and 1 minute break between each round. Sanda competition has a weight scale. The available methods stipulated in Sanda's attack techniques are all kinds of martial arts moves other than the banned moves. Seemingly it is very open, all kinds of martial arts moves can be used, but the rules also stipulates the banned moves: The first rule is to strictly limit to use attack moves on head, elbow, knee and back joints and others to each other. Sanda has strict rules on the banned parts and scored parts. Sanda competitions also have detailed provisions on the scoring criteria, it is divided into: advantage victory, 3 points, 2 points, 1 point, no score, etc. Based on the different techniques used by the athlete, the produced effects for hit on different positions, the referees recorded player's scores in time according to the rules. In a formal Sanda competition, there is one referee on the court who is responsible for controlling the game, such as stop the competition in time, judging which side is on the ground and out of bounds, which side scores and how many scores to get, warn or advise player, announces which side wins, etc. . The judging content is a lot, so it is very difficult to operate. In Sanda competitions, there are generally 3 or 5 linesmen who are responsible for recording the scores of both sides, and determine which side wins in each round. Because there are many rules for scoring methods, scored points, scored positions, so the workload of the lineman is also very large and it is very professional. It is necessary to be trained to be competent for this task.

\section{CONCLUSION}

Sanda is mainly a technical sport which uses Chinese traditional martial arts, flexible and changeable moves and expressed in various forms, and kickboxing is a two-antagonist project, in the game the quality of the technology is on one hand, the power is also the key to victory. Boxing can effectively and accurately strike the effective part of the opponent and ensure that oneself is not hit is the key to boxing sports. These two completely different kinds of sports show 
the broad and profound Chinese traditional culture and Chinese characteristics of Sanda culture from the side, and also show the national characteristics of Western culture.

From the perspective of modern physical fitness, frequent practice of sanda and boxing competition can both improve people's strength and endurance physical fitness. Chinese Sanda sport can make better exercise on people's balance ability and improve the function of respiratory circulatory system, as well as people's flexibility.

\section{SUGGESTIONS}

Chinese Sanda must comply with the needs of the development of the times, on one hand, it must embody the national characteristics of Chinese Sanda, on the one hand, it must also constantly broaden its horizons, emancipate the mind, and absorb and draw on the advantages of similar projects at home and abroad, take the essence and discard the dregs, strengthen the construction of our own cultural concept while building our own national characteristics,.

In the form of protective gear, clothing, and venues, Chinese Sanda shall appropriately reform under the precondition of inheriting the excellent Chinese traditional sports spiritual systematic culture. For example: using finger gloves, it is easy to use a variety of ways to take and wrestle. It could make better reflection of the traditional techniques and national traditions of Chinese martial arts while enriching the attack techniques. In the design of clothing and venues it should reflect a distinctive national characteristic, so that the Sanda clothing and venues will be both good-looking and practical as well. In addition, commercial behaviors can be incorporated into clothing and venues, and advertisements can be placed on clothing and venues.

Chinese Sanda shall inherit and carry forward the precious traditional techniques of our country. It shall absorb and learn from the world's various excellent techniques and experiences in combat, including domestic wrestling, various types of martial arts, American MMA, Thai boxing, Taekwondo, kickboxing, karate, judo and other fighting techniques. Only through the extensive and comprehensive learning, we could be able to establish a relatively complete technical system based on kicking and throwing. Chinese Sanda must add elbow knees, large joints, and tricks. On one hand his will further improve the technical and tactical level and appreciation of Sanda, so that our Chinese martial arts can be counter-attacked against the American MMA, on the other hand it is also to further tap and highlight the essence of Chinese martial arts and better reflect the cultural characteristics of Chinese martial arts and spirit.

\section{CONCLUSION}

From the above historical context of China and South Korea, Confucius Ceremony Musical Dance experienced flourishing and declining together with the national fate, it is shouldering the important task to carry Dao through "dance". Although the dance performed at the Confucius ceremonies in China and South Korea is hard to find the appearance of the ancient system of the Three Generations, through the development track of Confucius Ceremony Musical Dance in the past dynasties, it is hoped that this will lay a solid theoretical foundation for the restoration of the modern Confucius Ceremony Musical Dance.

\section{REFERENCES}

[1] Liu Shujun. The philosophical thinking on the difference between national culture and the internationalized development of Martial Arts [J].Journal of Shanghai University of Sport, 2004(2).

[2] Tang Bo.Comparative Study on Competitive Martial Arts and Olympic Sports on the cultural background [J].Sports Culture Guide, 2002(3).

[3] Tang Bo, Lin Xiaomei. Comparative Study of Competitive Martial Arts and the Olympic Sports on the Culture Background $[\mathrm{J}]$. Sports Culture Guide. 2003 (03)

[4] Li Zhenjing, Wen Minghua, Lu Jun. Reviewing the Formation and Development of Sanda from the Integration and Conflict of Chinese and Western Sports Cultures [J] Boxing (Martial Arts Science).2010(07)

[5] Zhang Wentian, Liu Mingliang. Comparative Study of Sports Culture between Chinese Sanda and Western Boxing [J]. Journal of International Relations Institute. 2007(01)

[6] Chen Liang. Reviewing Sanda's Attack and Defense Concept from the Perspective of Historical Culture[J]. Journal of Guangzhou sports university 2008(06)

[7] Lin Xiaomei. Martial Arts enters the Olympic Games and docks between Chinese and Western cultures [J]. Sports Science. 2005(07)

[8] Zhang Xinping. The interpretation of the relationship between the American spirit and sports culture [J]. Sports Culture Guide. 2004(12) 